\title{
Multiscale sensorless adaptive optics OCT angiography system for in vivo human retinal imaging
}

Myeong Jin Ju

Morgan Heisler

Daniel Wahl

Yifan Jian

Marinko V. Sarunic 


\title{
Multiscale sensorless adaptive optics OCT angiography system for in vivo human retinal imaging
}

\author{
Myeong Jin Ju, ${ }^{*}{ }^{\dagger}$ Morgan Heisler, ${ }^{\dagger}$ Daniel Wahl, Yifan Jian, and Marinko V. Sarunic \\ Simon Fraser University, Department of Engineering Science, Burnaby, British Columbia, Canada
}

\begin{abstract}
We present a multiscale sensorless adaptive optics (SAO) OCT system capable of imaging retinal structure and vasculature with various fields-of-view (FOV) and resolutions. Using a single deformable mirror and exploiting the polarization properties of light, the SAO-OCT-A was implemented in a compact and easy to operate system. With the ability to adjust the beam diameter at the pupil, retinal imaging was demonstrated at two different numerical apertures with the same system. The general morphological structure and retinal vasculature could be observed with a few tens of micrometer-scale lateral resolution with conventional OCT and OCT-A scanning protocols with a 1.7-mm-diameter beam incident at the pupil and a large FOV $(15 \mathrm{deg} \times 15 \mathrm{deg})$. Changing the system to a higher numerical aperture with a 5.0-mm-diameter beam incident at the pupil and the SAO aberration correction, the FOV was reduced to $3 \mathrm{deg} \times 3 \mathrm{deg}$ for fine detailed imaging of morphological structure and microvasculature such as the photoreceptor mosaic and capillaries. Multiscale functional SAOOCT imaging was performed on four healthy subjects, demonstrating its functionality and potential for clinical utility. ๑ 2017 Society of Photo-Optical Instrumentation Engineers (SPIE) [DOI: 10.1117/1.JBO.22.12.121703]
\end{abstract}

Keywords: optical coherence tomography; adaptive optics; angiography; retina.

Paper 170511SSR received Aug. 2, 2017; accepted for publication Oct. 5, 2017; published online Nov. 1, 2017.

\section{Introduction}

Optical coherence tomography (OCT) ${ }^{1}$ has become an invaluable imaging modality with widespread biomedical applications, especially in ophthalmology.,3 The eye is arguably the most optically accessible organ of the human body, allowing OCT to be used for measurements of the retinal structure, ${ }^{4}$ which is useful for the diagnosis and monitoring the progression of conditions such as age-related macular degeneration, diabetic retinopathy, and glaucoma. ${ }^{2}$

One of the attractive characteristics of OCT is the decoupling of axial (depth) and transverse (lateral) resolutions. The transverse resolution is determined by the numerical aperture (NA) of the optical system while the axial resolution relies on the spectrum of the light source (center wavelength and 3-dB bandwidth). While OCT permits independent control of its lateral and axial resolutions, an increased NA limits the confocal depth of the system, which affects the image quality at depths outside the beam waist. With an axial resolution of 2 to $15 \mu \mathrm{m}$ determined by the source parameters, it is possible to achieve volumetric imaging with a millimeter-scale imaging depth and a few tens of micrometer transverse resolution with a low NA objective lens or, in an alternative configuration, a few tenths of a millimeter-scale imaging depth and a few micrometer transverse resolution with a high NA objective lens. In the case of human retinal imaging, the combined cornea and intraocular lens act as the objective lens, so the maximum achievable transverse resolution on the retina is related to the diameter of the pupil. In general, a narrow beam, about 1 to $2 \mathrm{~mm}$ in diameter, is employed for common commercially available retinal diagnostic OCT systems, which results in a spot size on the retina of around $25 \mu \mathrm{m}$ in diameter. To achieve a smaller spot size on

\footnotetext{
*Address all correspondence to: Myeong Jin Ju, E-mail: mjju@ @fu.ca
}

${ }^{\dagger}$ Cofirst authors of this paper. the retina, down to only a few micrometers, the diameter of the imaging beam incident on the cornea can be increased up to $8 \mathrm{~mm}$ at the dilated pupil, so the maximum NA of the human eye of about 0.23 can be achieved. However, with the use of such a high NA, the optical aberrations induced by the eye increase as well.

Adaptive optics (AO), which was originally introduced for astronomy to sharpen the images acquired of the stars, ${ }^{5}$ has been successfully integrated with OCT to enable diffraction limited imaging performance for retinal applications. ${ }^{6-12}$ A few recent reviews have been published on $\mathrm{AO}$ in combination with OCT. ${ }^{13-16}$ In addition to the visualization of the photoreceptor mosaics, AO-OCT has been demonstrated for OCTangiography (OCT-A) of the retinal circulation, ${ }^{17}$ as well as visualization of the choriocapillaris. ${ }^{18,19}$ In a conventional AO-OCT system, wavefront distortion caused by the human eye can be measured by a wavefront sensor and corrected with deformable optical elements. The wavefront measurement is commonly performed using a Hartmann-Shack wavefront sensor (HS-WFS) placed at a plane that is optically conjugated to the pupil of the eye. The deformable optical element, such as a deformable mirror, is placed at an optical plane that is conjugated to the pupil and to the HS-WFS. Due to the high sensitivity of the HS-WFS to the back-reflections, the optical system is commonly implemented with concave mirrors instead of lenses. In addition, long focal length concave mirrors are generally used to suppress the off-axis aberrations. ${ }^{10,20,21}$ These specific requirements can consequently make AO-based ophthalmic imaging systems difficult for practical use in a clinical environment. ${ }^{22}$ In terms of retinal imaging field-of-view (FOV), while a high resolution of $\sim 3 \mu \mathrm{m}$ can be achieved over an area of $1.5 \mathrm{deg} \times 1.5 \mathrm{deg}(450 \mu \mathrm{m} \times 450 \mu \mathrm{m})$, in a clinic the desired FOV generally tends to be much larger; for example,

$1083-3668 / 2017 / \$ 25.00$ (c) 2017 SPIE 
$\sim 20 \mathrm{deg} \times 20 \mathrm{deg}$ is the standard for an OCT macular cube analysis.

Recently, we reported a lens-based SAO-OCT retinal imaging system with potential to meet clinical retinal imaging needs. ${ }^{23}$ Instead of adopting the HS-WFS for measuring the wavefront aberrations, wavefront sensorless adaptive optics (SAO) optimization was used to drive the shape of the wavefront correcting elements based on an image quality metric. Two separate deformable lens components, a variable focus lens and a multiactuator adaptive lens were utilized to correct the aberration up to fourth radial-order Zernike polynomials. A major benefit of the SAO algorithm with the transmissive deformable lenses was that the system design could be compatible with a typical lens-based OCT system in an optical setup with a small footprint. However, the available stroke and settling time of the deformable elements limited the aberration correction performance and speed.

Since reliable aberration correction performance and speed are key elements for clinical imaging studies, we developed a new generation of SAO-OCT with a high-stroke and a highspeed deformable mirror. Based on the specifications provided by the manufacturer, it has 69 -actuators, $60-\mu \mathrm{m}$ stroke, and $800-\mu$ s settling time with less than $3 \%$ nonlinear error. Using the polarization properties of light, the system was designed to fit in a small footprint (12 in. $\times 12$ in.) without compromising imaging performance. In addition, by employing a zoomable collimator, the diameter of the imaging beam delivered to the pupil could be adjusted from 1.7 to $5.0 \mathrm{~mm}$ without any modification to the optical layout. This adjustable pupil size capability permitted the option to choose the use of the system for conventional OCT imaging (long imaging depth with a low lateral resolution) or for high-resolution SAO-OCT imaging (short imaging depth with a high lateral resolution). By adopting a multiple B-scan (BM-scan) protocol, vasculature specific contrast imaging often referred to as was also performed. Owing to the dual modalities, two different OCT-A imaging modes could be performed and compared, one with conventional
OCT and another with SAO-OCT, using the same system. In this paper, we demonstrate the benefit of a multiscale SAOOCT for in vivo human retinal imaging.

\section{SAO-OCT-A System}

An overall schematic of the SAO-OCT-A system used in this study is shown in Fig. 1(a); it is an improved version of a previously reported SAO-OCT system. ${ }^{23}$ In the system, a MEMSbased swept source with a $200-\mathrm{kHz}$ sweep rate (Axsun Technology Inc.), a duty cycle of $78 \%$, a center wavelength of $1.06 \mu \mathrm{m}$, and a spectral range of $\sim 110 \mathrm{~nm}$ was employed. There are two interferometers in the system schematic: a phase stabilization unit (green fiber coupler) and the SAO-OCT interferometer (blue fiber coupler). The phase stabilization was a fiber-based Michelson interferometer that generated a fixed interference signal referred to as the calibration signal, with dispersion mismatch minimized using identical optical components. In the sample arm [shown in Fig. 1(b)], the probe beam from a zoomable collimator (AC618APC-B, Thorlabs Inc.) directed the light to a polarizing beam splitter (PBS), and the P-polarization beam passed through the PBS while the S-polarization light was reflected. Here, it is noted that only the P-polarization was used for OCT imaging. By adjusting a polarization controller before the collimator, the optical powers of two orthogonal polarization states were controlled to maximize the light in the P-polarization. After passing through the PBS, the polarization plane of the P-polarization was rotated by 90 deg using an achromatic zero-order quarter-wave plate (QWP) oriented at $45 \mathrm{deg}$, reflected by the deformable mirror (DM; DM-69, ALPAO, France), and reverse passed through the QWP. This 90 deg polarization plane rotated beam ( $S$-polarization beam) was reflected by the PBS and directed to the rest of the optics in the sample arm. The OCT and calibration signals were detected by the same balanced photodetector (Model 1817, Newport Corp.) and digitized by a ATS9350 analog-to-digital converter (AlazarTech, Canada) with a sampling rate of $500 \mathrm{MHz}$. To achieve a phase-resolved OCT signal, the spectral
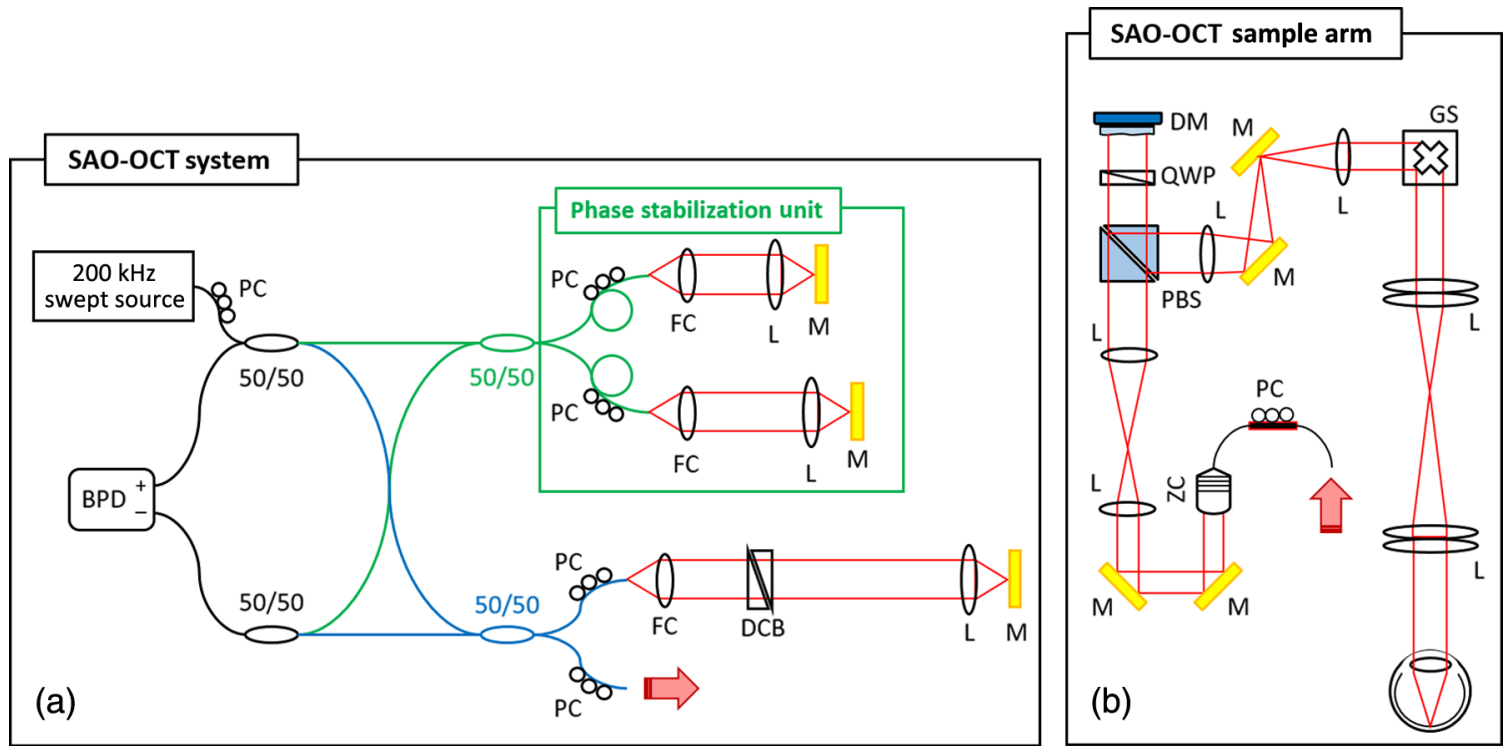

Fig. 1 Schematic of the (a) SAO-OCT system and (b) SAO-OCT sample arm. L, lens; M, mirror; FC, fiber collimator; PC, polarization controller; $\mathrm{BPD}$, balanced photodetector; $\mathrm{DCB}$, dispersion compensation block; PBS, polarization beam splitter; QWP, quarter wave plate; ZC, zoomable collimator; GS, galvanometer scanner. 
shift caused by fluctuation in the synchronization between the wavelength sweep of the light source and the digitization was estimated by cross-correlation of the calibration signals from subsequent A-scans and corrected by resampling the spectrum based on the estimation result. ${ }^{24,25}$ With the spectral sampling parameter and average probing power of $900 \mu \mathrm{W}$, the depth resolution defined by the $-6 \mathrm{~dB}$ width was measured to be $11 \mu \mathrm{m}$ in air [corresponding to a resolution of $8.0 \mu \mathrm{m}$ in tissue $(n=1.38)$ ], and the sensitivity was determined to be $99.1 \mathrm{~dB}$ with the signal roll-off of $-1.05 \mathrm{~dB} / \mathrm{mm}$ measured at a depth range of 0.3 to $2.5 \mathrm{~mm}$. There are two different imaging NA settings: low NA and high NA with a $1.7-$ and $5.0-\mathrm{mm}$ beam diameter at the cornea, respectively. Assuming a 22.2-mm focal length of the eye and refractive index of 1.33 for water at $1.06 \mu \mathrm{m}$, the transverse resolution was estimated to be nominally 10.6 and $3.6 \mu \mathrm{m}$ with the low and high NAs, respectively, as defined by $\Delta x_{\mathrm{OCT}}=0.51 \lambda / \mathrm{NA}^{14}$

\section{Imaging Protocols}

Retinal imaging was performed with the probe beam power of $\sim 900 \mu \mathrm{W}$ at the cornea below the ANSI maximum ocular permissible exposure limit ${ }^{26}$ and was measured prior to imaging to be below $900 \mu \mathrm{W}$. To navigate to the region of interest (ROI) on the retina, the system was operated in widefield mode with a transverse area of $15 \mathrm{deg} \times 15 \mathrm{deg}[4.5 \mathrm{~mm} \times 4.5 \mathrm{~mm}$; shown in Fig. 2(a)] scanned with $300 \times 300$ A-lines using a $1.7-\mathrm{mm}$ beam diameter at the pupil (low NA setting). After manually aligning to the ROI, the transverse area was changed to $6 \mathrm{deg} \times 6 \mathrm{deg}[1.8 \mathrm{~mm} \times 1.8 \mathrm{~mm}$; shown in Fig. 2(b)] to avoid undersampling with the same $300 \times 300$ A-scans sampling density (pixel separation of $6 \mu \mathrm{m}$ versus $1 / \mathrm{e}^{2}$ Gaussian beam waist of $\sim 12 \mu \mathrm{m}$ in the retina). For OCT-A imaging, three multiple B-scans (constituting a BM-scan) were taken at the same location on the retina and the speckle variance calculation ${ }^{27}$ was applied. Since the major aberration when imaging with the low NA setting is defocus, the DM was used only for manual defocus correction based on qualitative image brightness, particularly at the outer retina. The imaging resolution was increased to the high NA setting by adjusting the beam size at the zoomable collimator. The same sampling protocol was used for the high-resolution mode, but the scanning dimension was decreased to $3 \operatorname{deg} \times 3 \operatorname{deg}[900 \mu \mathrm{m} \times 900 \mu \mathrm{m}$; shown in Fig. 2(c)]. For the high-resolution mode with $5.0-\mathrm{mm}$ beam diameter, a single drop of $1 \%$ tropicamide (Mydricayl) was applied for inducing dilation of the pupil. During high-resolution imaging, GPU-based real-time retinal axial tracking was applied on the B-scan image and the SAO algorithm was applied to the en face retinal layer image at the ROI depth. ${ }^{28,29}$ For OCTA imaging with the two different OCT imaging modes, a three BM-scan protocol was employed, so the total scanning density changed to $300 \times 900(300 \times 3)$ A-lines. OCT-A imaging was performed using a GPU-based real-time acquisition and display preview of the angiography. ${ }^{30}$ To demonstrate the functionality and clinical utility of the system, the posterior eye of human volunteers without any remarkable retinal disorder was imaged. All protocols for measurement were approved by the Office for Research Ethics at Simon Fraser University. Written and informed consent was obtained prior to imaging.

\section{Sensorless Adaptive Optics Optimization Algorithm}

The SAO algorithm used in this work is similar to our previous reports on SAO. ${ }^{23,31,32}$ Owing to the increased stroke of the deformable mirror (AlpAO) compared with the previous system, only a single wavefront controlling element was incorporated in the SAO-OCT system to correct the aberrations up to fourthradial order Zernike polynomials. At the beginning of the aberration correction process, the imaging FOV was reduced to $1 \mathrm{deg} \times 1 \mathrm{deg}(\sim 300 \mu \mathrm{m} \times 300 \mu \mathrm{m}$ on the retina), which is close to the size of the isoplanatic patch, $\sim 1.1 \mathrm{deg} \times 1.1 \mathrm{deg}$ $(330 \mu \mathrm{m} \times 330 \mu \mathrm{m}){ }^{33}$ From an OCT volume acquired with this reduced FOV, an en face OCT image corresponding to the specific depth plane, or retinal layer of interest, was selected and extracted by real-time automated retinal tracking software. ${ }^{29}$ For each Zernike mode, the deformable mirror was stepped through the empirically determined range of coefficient values. At each step, the image quality metric was calculated as the summed intensity of the extracted en face image. In a hill-climbing

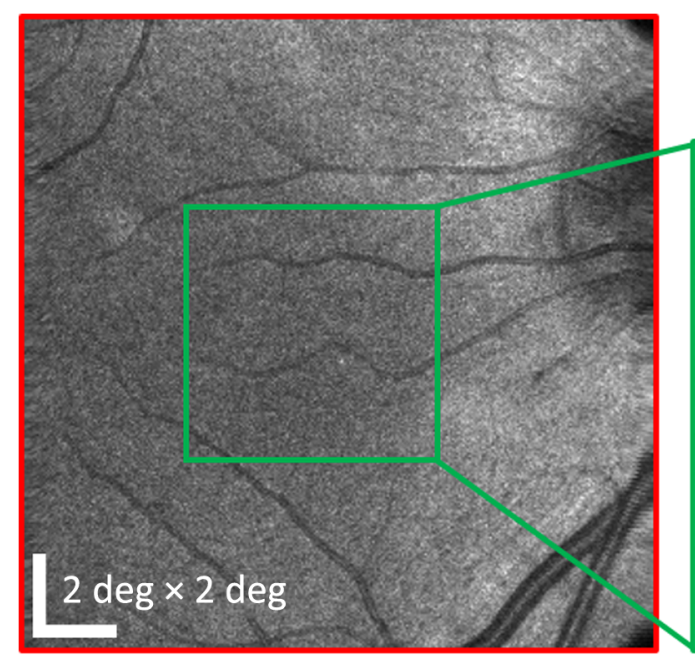

(a)

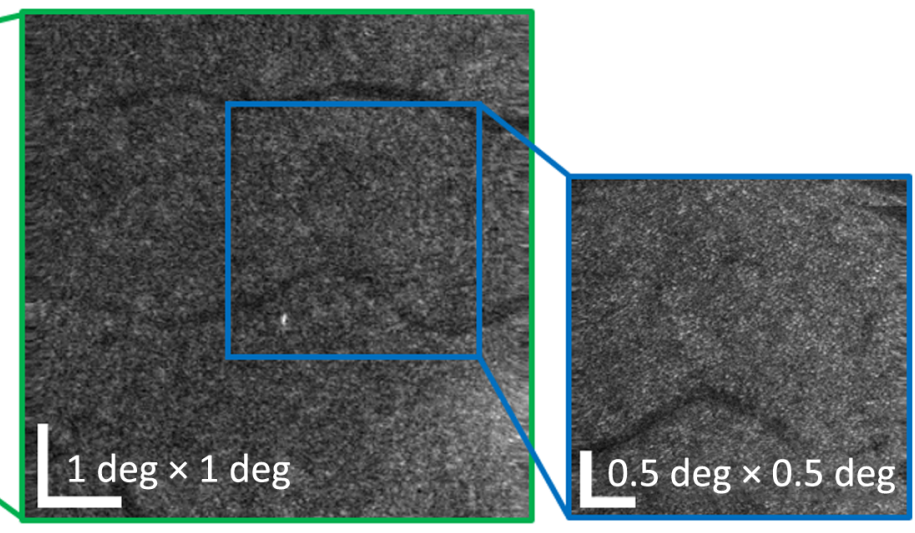

(b) (c)

Fig. 2 Multiple FOV OCT images. (a) 15 deg $\times 15$ deg FOV and (b) 6 deg $\times 6$ deg FOV with $1.7 \mathrm{~mm}$ beam incident on the cornea (low NA). (c) 3 deg $\times 3$ deg FOV with 5.0-mm beam incident on the cornea (high NA). Images acquired from the left eye of subject 1 (21-year-old healthy male). 


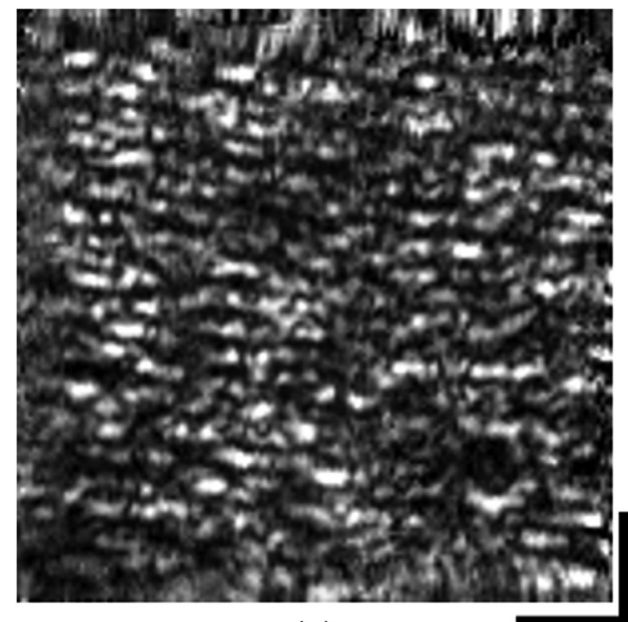

(a)

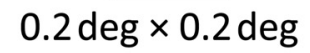

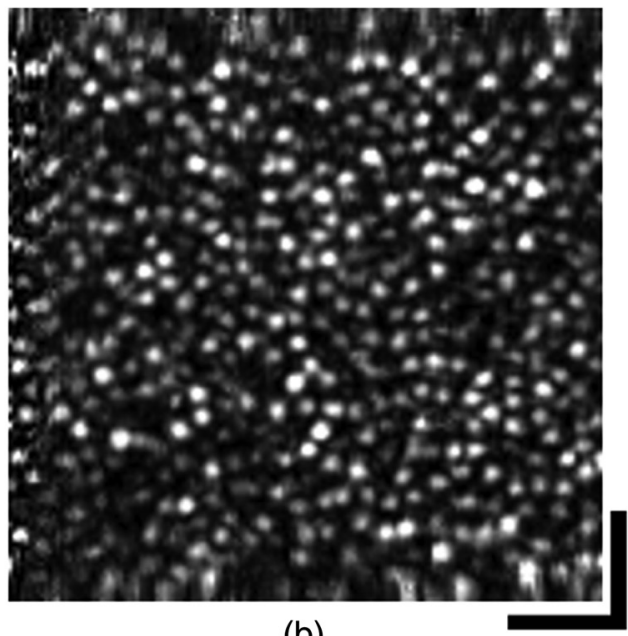

(b)

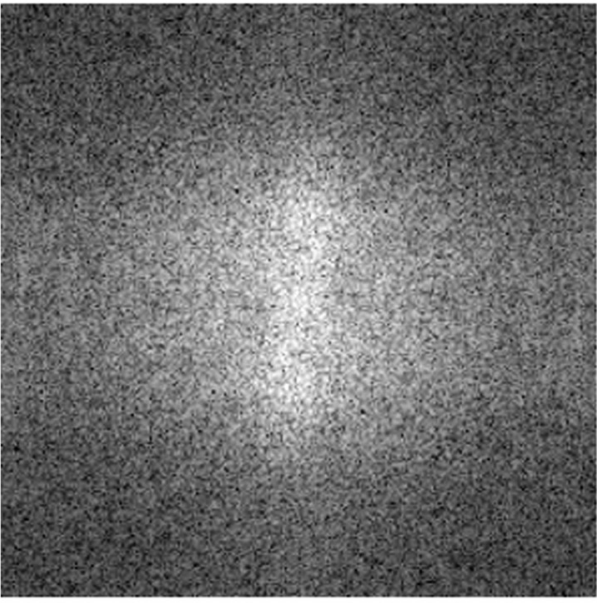

(c)

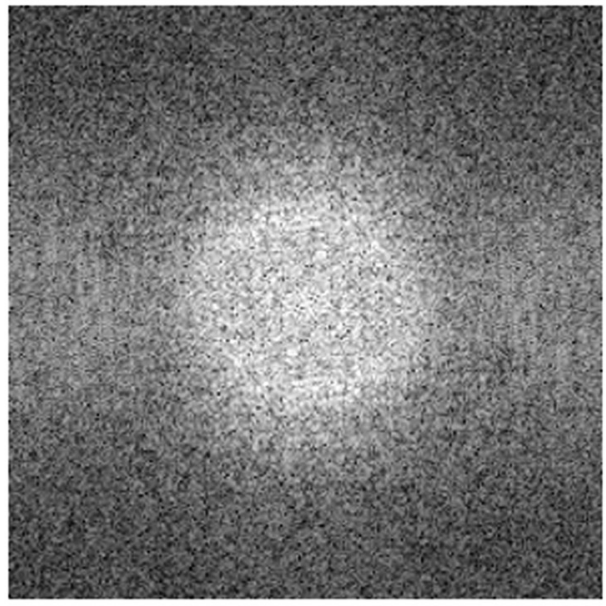

(d)

Fig. 3 The effects of the SAO optimization on the photoreceptor cone mosaic images. Images acquired with a 4-mm beam incident on the cornea (a) without SAO optimization and (b) with SAO optimization. The power spectrum of the images in (a) and (b) are shown in (c) and (d), respectively. The FOV of the image is $1 \mathrm{deg} \times 1 \mathrm{deg}(300 \mu \mathrm{m} \times 300 \mu \mathrm{m})$ taken at $\sim 4$ deg eccentricity from the fovea. Images acquired from the right eye of subject 2 (25-year-old healthy female).

fashion, the coefficient corresponding to the brightest image from the 21 uniformly sampled coefficient values was applied to the deformable mirror for each Zernike mode. The SAO optimization was performed at a rate of 200 frames per second with $100 \times 100$ A-scans, and its performance was tracked during the image acquisition by comparing the images before and after the optimization result. Figure 3 shows representative human photoreceptor cone mosaic images acquired with a 5.0-mm-diameter beam incident on the cornea before [Fig. 3(a)] and after the SAO aberration correction [Fig. 3(b)]. In addition, comparing the spatial density of the photoreceptor image before [Fig. 3(c)] and after [Fig. 3(d)] the SAO-based aberration correction, increased contributions at frequencies around Yellott's ring ${ }^{34}$ can be observed, which supports the image improvement after the SAO aberration correction.

\section{Results}

Figure 4 shows representative B-scan images acquired with (a) low NA $(1.7 \mathrm{~mm})$ and (b) high NA $(5.0 \mathrm{~mm})$ settings. For both $\mathrm{B}$-scan images, the focus was set at the photoreceptor layer. The line plot placed at the right side of each figure is the intensity plot averaged across the B-scan direction; within the yellow dashed box in Fig. 4(a) and for the entire B-scan in Fig. 4. From the comparison of the mean intensity profile plots in Figs. 4(a) and 4(b), a more prominent peak is observed at the photoreceptor layer where the focus was set in Fig. 4(b).

Figure 5 shows representative structural data of the retinal nerve fiber layer (RNFL) taken with the system at low NA, high NA, and high NA with SAO optimization. At the low NA setting, it is difficult to recognize individual nerve fiber bundles. After changing to the high NA setting, nerve fiber bundles can be observed with moderate contrast. After correcting the aberrations with the SAO algorithm, individual bundles are clearly visualized with high contrast.

Representative results for the multiscale imaging performance are shown in Fig. 6. Figures 6(a) and 6(b) show en face OCT images extracted from the photoreceptor layer in the $15 \operatorname{deg} \times 15 \mathrm{deg}$ FOV and $6 \operatorname{deg} \times 6 \mathrm{deg}$ FOV, respectively, acquired with the low NA setting without SAO optimization. From the en face images, general morphological features such as thick retinal vessels and the optic nerve head can be 


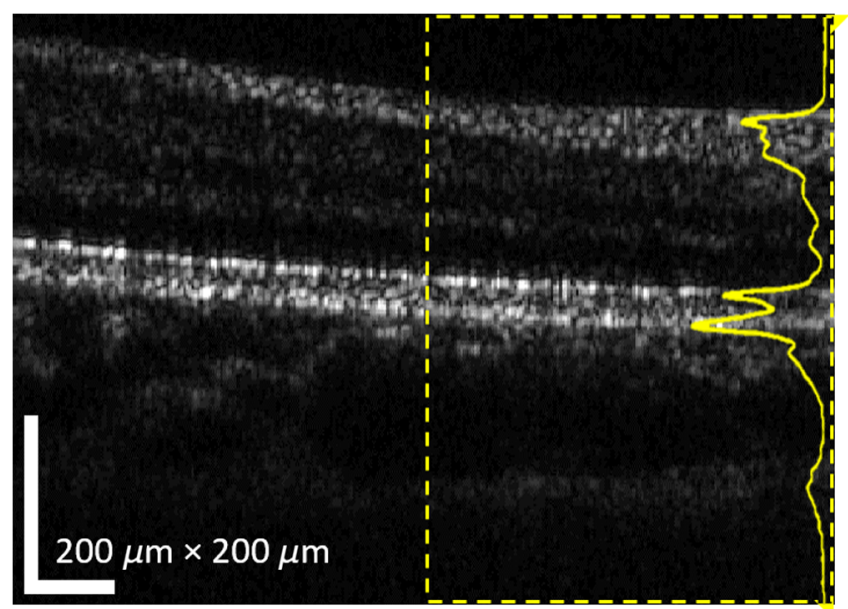

(a)

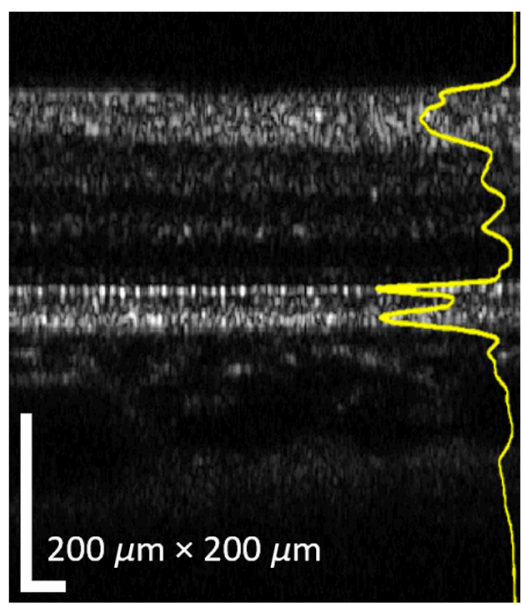

(b)

Fig. 4 B-scan images of (a) standard (low NA) OCT with a 1.7- $\mathrm{mm}$ beam diameter without SAO optimization and (b) high NA OCT with a 5.0-mm beam diameter incident on the cornea with SAO optimization. Images acquired from the right eye of subject 3 (31-year-old healthy female).

Low NA

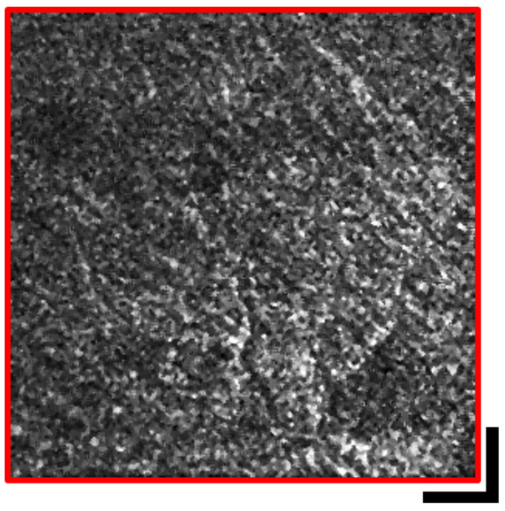

$1 \operatorname{deg} \times 1 \mathrm{deg}$

(a)

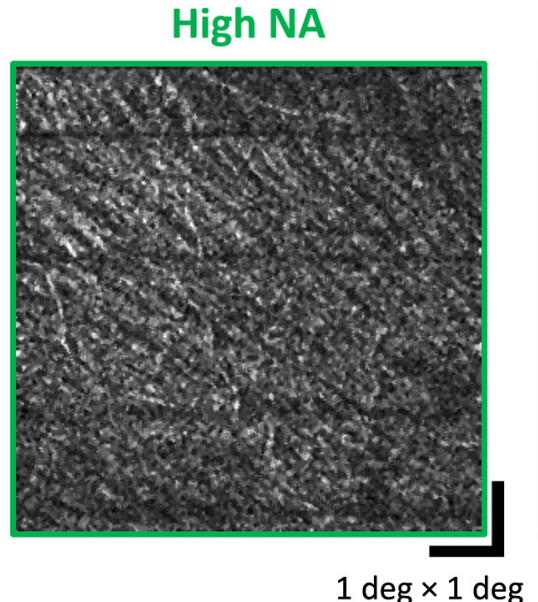

(b)

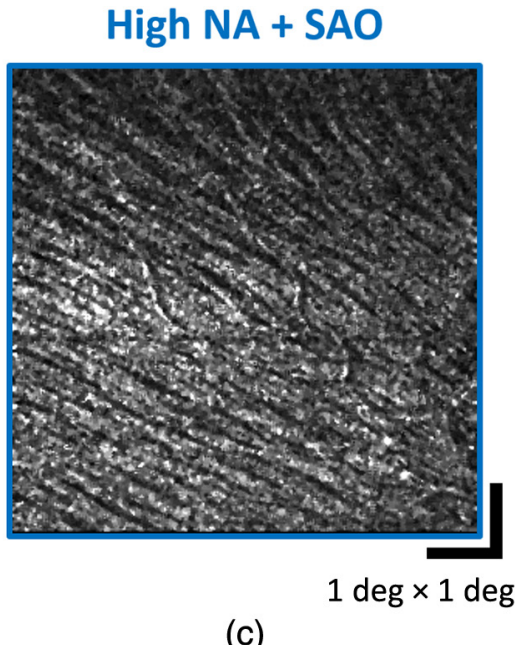

(c)

Fig. 5 En face images of the RFNL acquired at (a) low NA, (b) high NA, and (c) high NA with SAO optimization. Images acquired from the left eye of subject 4 (23-year-old healthy female).

observed. In some locations of Fig. 6(b), the well-defined circular pattern of the photoreceptor mosaic cannot be clearly visualized, instead random speckle pattern is predominantly observed. In contrast, Figs. 6(c) and 6(d) show the resolved photoreceptor cone mosaic images acquired with the 5.0-mmdiameter beam incident on the cornea corresponding to the (c) red, (d) green, and (e) blue boxes in Fig. 6. After the SAO optimization process, the photoreceptor cone mosaics were clearly resolved in these $3 \mathrm{deg} \times 3 \mathrm{deg}$ FOV images.

By calculating the OCT signal variance across each set of three BM-scans within the volumetric data, vasculature specific contrast can also be extracted from the same subject data set presented in Fig. 6, and it is shown in Fig. 7. From the angiography images with low NA (1.7 mm beam diameter), the overall retinal vasculature pattern can be observed in Fig. 7(a) while the capillaries in the outer plexiform layer (OPL) cannot be clearly visualized even in the zoomed-in $6 \operatorname{deg} \times 6 \operatorname{deg}$ FOV OCT-A image [Fig. 7(b)]. On the other hand, the capillaries are more clearly observed in the images acquired with $5.0-\mathrm{mm}$ beam diameter high-resolution imaging mode even though the focus was set at the outer retina [Figs. 7(c)-7(e)].

The cone photoreceptor mosaic was resolved after SAO aberration correction down to a retinal eccentricity of $\sim 2.5 \mathrm{deg}$ in all the subjects that were imaged. We note that AO is not strictly required to visualize the photoreceptors, particularly at large retinal eccentricities and for young healthy subjects. Closer to fovea, and especially in clinical populations, $\mathrm{AO}$ is essential to achieving an adequately small focal spot to reveal the mosaic pattern. In this report, we used a relatively long wavelength for imaging $(1.06 \mu \mathrm{m})$ and a 5.0-mm beam to have the long image depth and sweep rates afforded by swept source OCT and better transmission through potentially cloudy corneas and intraocular lenses that are common in clinical populations. Figure 8 shows representative multiscale functional OCT images taken close to the fovea with the high NA imaging mode. From the 15 deg $\times 15$ deg FOV images [Fig. 8(a)], the general morphological features and vascular patterns can be observed. Interestingly, we can still generally visualize the circular shape 


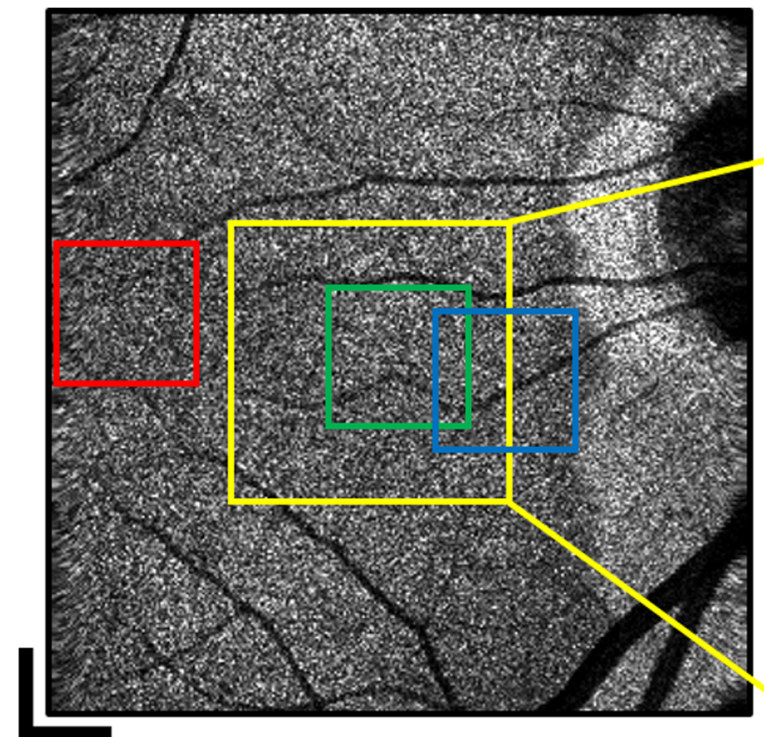

$2 \operatorname{deg} \times 2$ deg

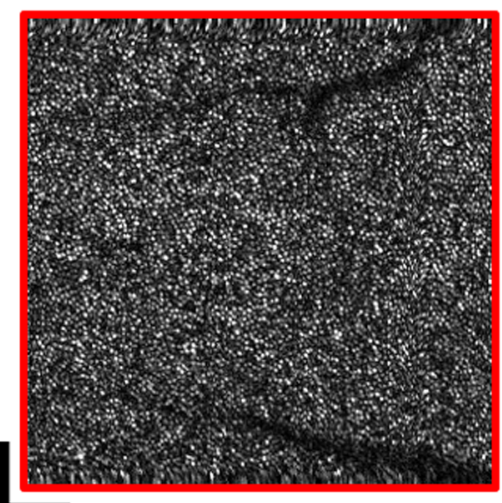

$0.5 \mathrm{deg} \times 0.5 \mathrm{deg}$ (c) (a)

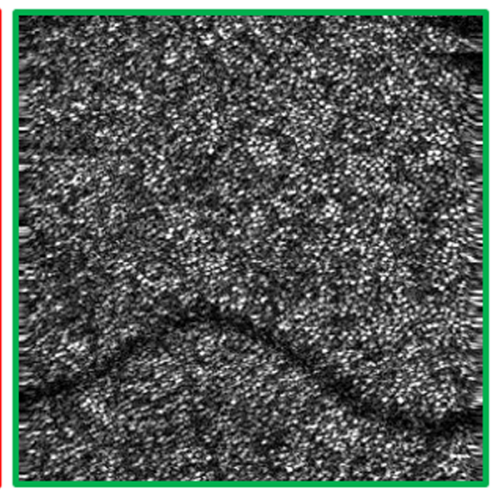

(d)
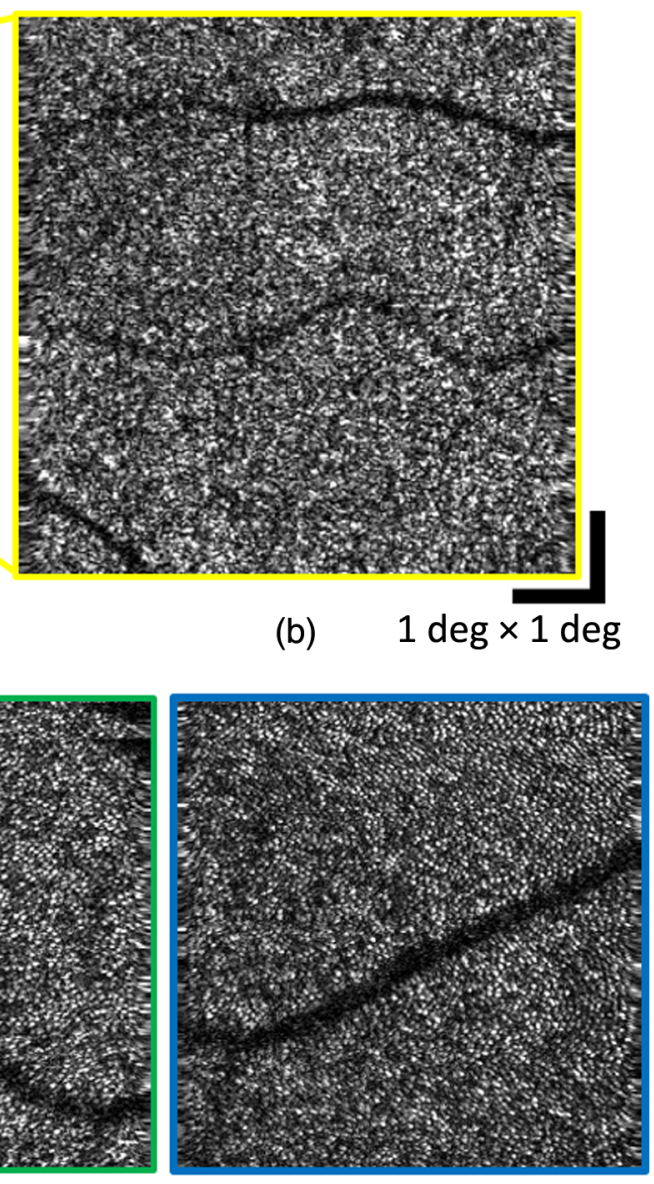

(e)

Fig. 6 Representative multiscale photoreceptor imaging. (a) 15 deg $\times 15$ deg FOV standard OCT without SAO optimization, (b) $6 \mathrm{deg} \times 6 \mathrm{deg}$ FOV standard OCT without SAO optimization, (c-e) 3 deg $\times 3$ deg FOV high-resolution OCT with SAO optimization at different locations marked by (c) red, (d) green, and (e) blue boxes in (a). (b) Image was acquired at 4.5 deg to 10.5 deg eccentricity from the fovea. Images acquired from the left eye of subject 1 (21-year-old healthy male).

of photoreceptors and thin capillaries even with the undersampled condition in the $6 \operatorname{deg} \times 6$ deg FOV images [Fig. 8(b)]. Within the $3 \mathrm{deg} \times 3 \mathrm{deg}$ FOV images [Fig. 8(c)], the wellresolved photoreceptor and more defined capillaries are observed.

The high sensitivity of OCT detection allowed for the full thickness of the retina to be readily observable, even the layers well outside the Rayleigh range of the focal position on the outer retina. When imaging with high NA focused on the photoreceptors, the imaging probe diameter at the inner retina is significantly larger due to beam divergence. Hence, for the SAOOCT-A images presented here, the appearance of the capillaries is not attainable at the full resolution. Despite the location of the focus at the outer retina, the deep capillary layer (capillaries in the OPL) was readily observable with good contrast, as presented in Fig. 7. In comparison, when imaging with the low NA settings, the deep capillary layer had lower contrast. As a potential site of early change in diabetic retinopathy, the ability to visualize the deep capillary layer is of particular interest. ${ }^{35,36}$
In addition, by adjusting the focal position to the RNFL, the radial peripapillary capillaries (RPCs) can also be visualized. The RPCs are typically extremely long thin capillaries with little variation in caliber and a radiating structure oriented parallel to the NFL axons. The RPCs represent an additional plexus layer that is of interest to clinicians. Although there exists evidence in the Literature that RPCs are critically related to retinal ganglion cell (RGC) function ${ }^{37-39}$ and time-dependent loss of which is characteristic of glaucoma, ${ }^{40}$ they are not commonly assessed in the clinic. This may be due to the inability to reliably visualize RPCs with fluorescence angiography, ${ }^{41}$ which is the clinical standard for evaluating the retinal microvasculature, although it may become more popular as the RPCs can be assessed using the standard OCT-A. ${ }^{42}$ As shown in Fig. 9, we can visualize this unique plexus using the SAO-OCT-A system by utilizing the zoomable collimator for high NA imaging. For future studies, the use of the strip-based registration technique ${ }^{43}$ could be used to enhance our OCT-A quantitative analyses. ${ }^{44}$ 


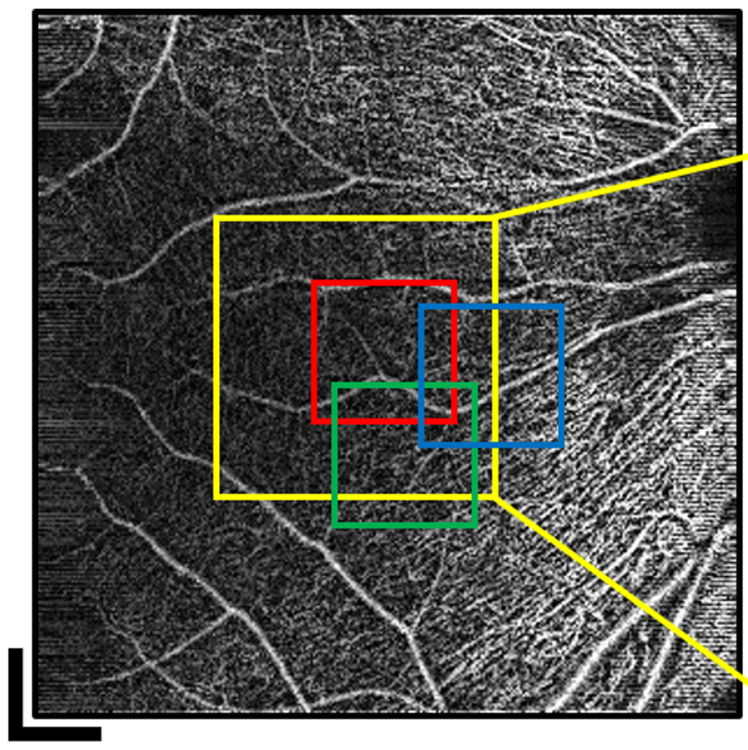

2 deg $\times 2$ deg

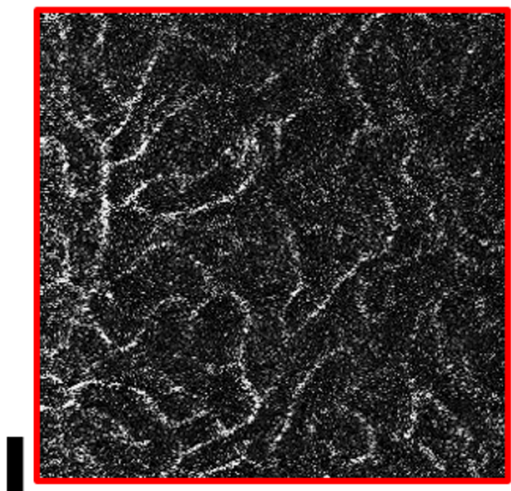

$0.5 \mathrm{deg} \times 0.5 \mathrm{deg}$ (c) (a)

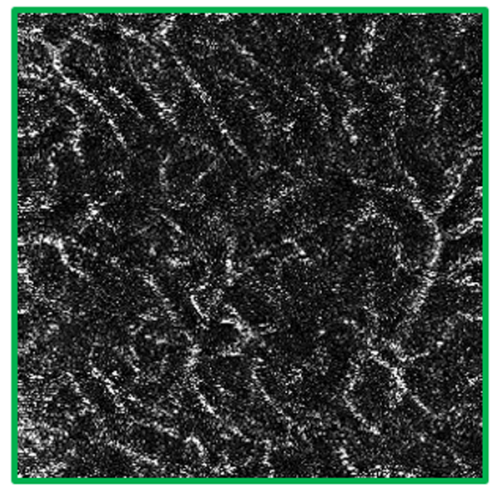

(d)

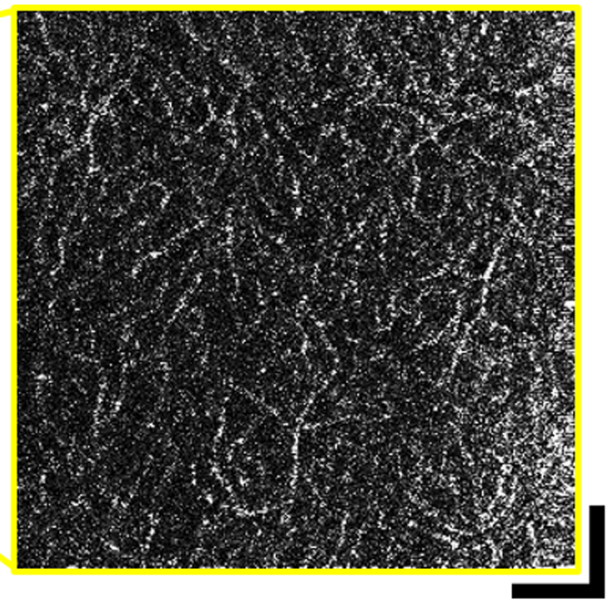

(b) $1 \mathrm{deg} \times 1 \mathrm{deg}$

Fig. 7 Representative multiscale OCT-A imaging set from the same subject shown in Fig. 6 . (a) $15 \mathrm{deg} \times 15 \mathrm{deg}$ FOV retinal vasculature image and (b) 6 deg $\times 6$ deg FOV capillaries imaged with a 1.7-mm beam incident on the cornea. (c-e) Zoomed-in high resolution images of capillaries acquired with a 5.0-mm beam incident on the cornea with a $3 \mathrm{deg} \times 3 \mathrm{deg}$ FOV at the different locations as indicated by the (c) red, (d) green, and (e) blue boxes in (a). Images acquired from the left eye of subject 1 (21-year-old healthy male).

\section{Discussion}

In this report, we presented a design of an SAO-OCT-A imaging system. By manipulating the polarization axis of the sample beam with a PBS and QWP, we were able to design a lensbased light delivery path and minimize the overall system size. At the beginning of the beam path in the sample arm, the $P$-polarization transmitted through the PBS was selected for OCT imaging due to the high extinction ratio of the transmission axis of the PBS. ${ }^{25}$ Another unique aspect of our system is the capability to change the effective imaging NA. Through the use of a zoomable collimator, the diameter of the probe beam delivered to the cornea was controlled without variation in the optical power, which allows the system to maintain its sensitivity with different imaging NA settings. At the low NA setting, the imaging system performance was similar to conventional OCT and OCT-A systems. ${ }^{42,43}$ At the high NA setting, with a 5.0-mm-diameter beam incident on the cornea and with the help of the SAO optimization algorithm, the resolution at the retina was improved enough to resolve the photoreceptor mosaic, which is not attainable with the low NA setting. The imaging protocol was set to first align and image with the widefield mode, which allowed for easy identification of regions of interest; then after twisting the zoomable collimator (and thereby increasing the system NA), SAO aberration correction was performed to visualize the photoreceptor mosaic with cellular resolution.

In the widefield mode of operation, the FOV on the retina was $\sim 15 \mathrm{deg}$, which is smaller than the $6 \times 6 \mathrm{~mm}$ macular cube analysis that is commonly performed with commercial imaging systems. The design of the sample arm optics was performed using commercially available off-the-shelf lenses, and the desire for a wide FOV was balanced with the requirement for diffraction limited performance when imaging with a high NA in a narrower field. The widefield can be readily expanded without compromising the high NA imaging through the use of custom lens designs. 

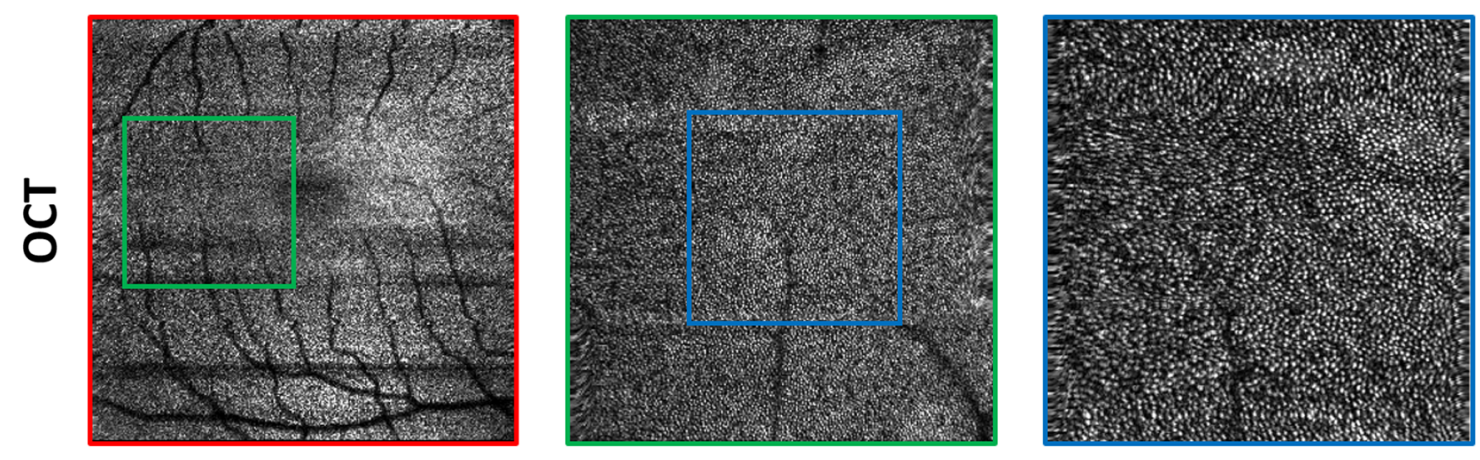

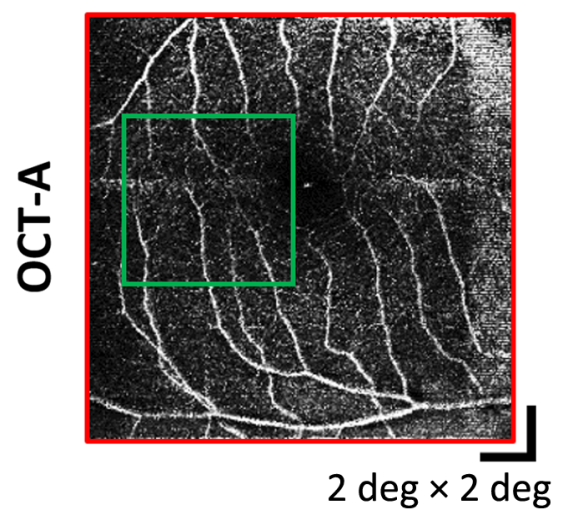

(a)

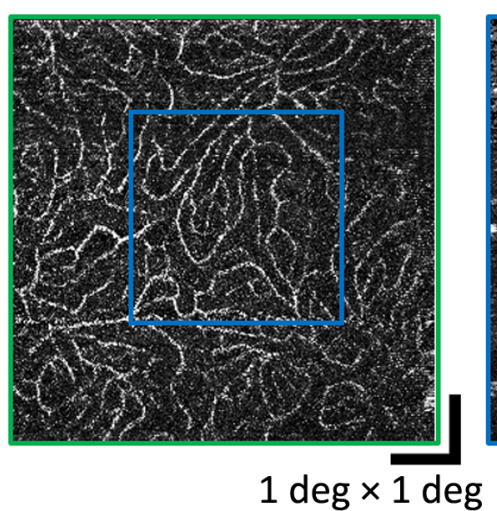

(b)

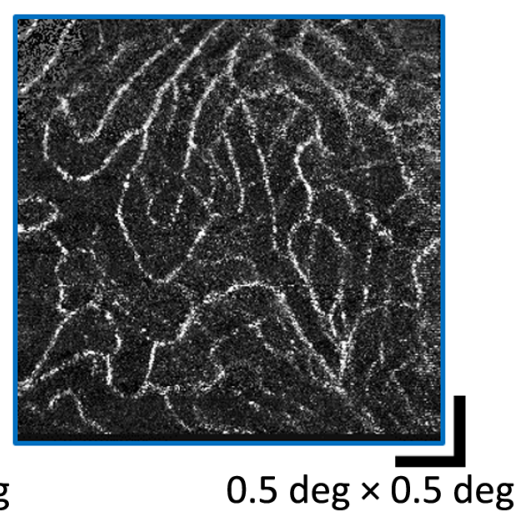

(c)

Fig. 8 Multiscale OCT and OCT-A imaging results with 5.0-mm beam diameter after aberration correction with the SAO optimization process. Top and bottom row images are en face OCT and OCT-A images. (a) $15 \mathrm{deg} \times 15 \mathrm{deg}$ FOV photoreceptor and retinal vasculature images. (b) and (c) $6 \operatorname{deg} \times 6$ deg FOV and 3 deg $\times 3$ deg FOV photoreceptor and OPL capillaries images, respectively. Images acquired from the left eye of subject 2 (25-year-old healthy female).

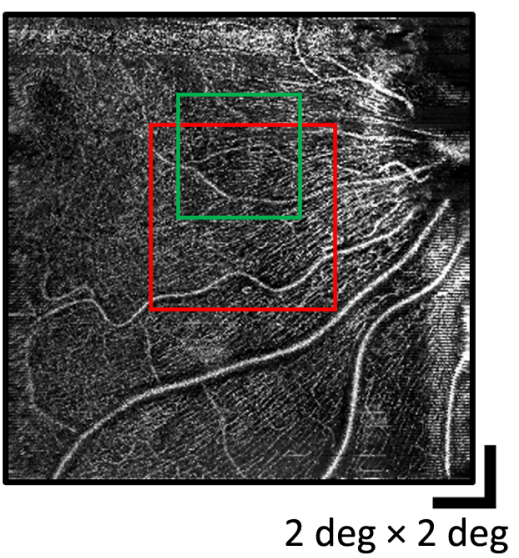

(a)

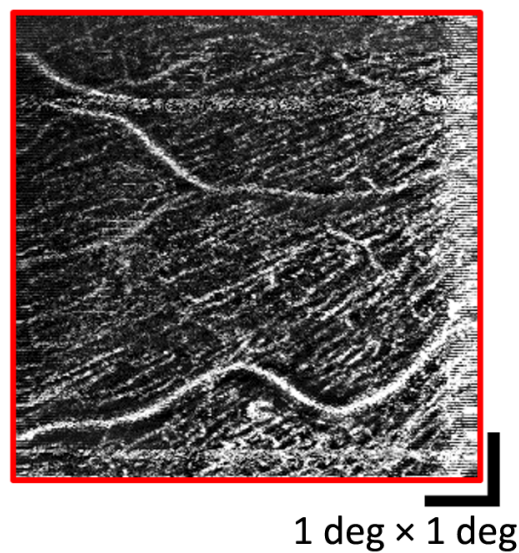

(b)

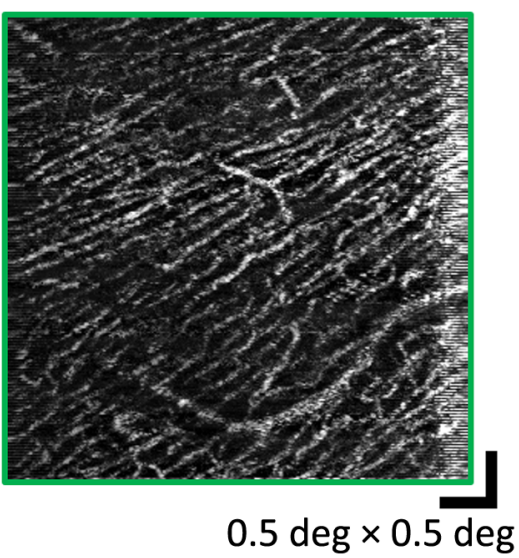

(c)

Fig. 9 Multiscale OCT-A imaging with the focal point set at the RFNL. (a) 15 deg $\times 15$ deg FOV retinal vasculature image and (b) 6 deg $\times 6$ deg FOV retinal vasculature and RPCs imaged with a $1.7-\mathrm{mm}$ beam incident on the cornea. (c) Zoomed-in high-resolution images of RPCs acquired with a $5.0-\mathrm{mm}$ beam incident on the cornea with a 3 deg $\times 3$ deg FOV at the location indicated by the green box in (a). Images acquired from the left eye of subject 4 (23-year-old healthy female).

Although images are not shown here, the zoomable collimator was continuously tunable and the probe beam diameter at the cornea could be set at any value between 1.7 and $5.0 \mathrm{~mm}$. For example, imaging with a beam of $\sim 3.0 \mathrm{~mm}$ would be useful in some applications, such as the optic nerve head. ${ }^{45}$ However, with the zoomable collimator adjusted at values below the maximum beam diameter, the light incident on the deformable aperture would not fill the aperture. Consequently, the wavefront correction applied by the deformable mirror would be inaccurate aside from defocus. For specific beam sizes less than the maximum, a distinct influence matrix could be generated considering only the central actuators on the deformable mirror. For example, ocular imaging with a beam size of $3 \mathrm{~mm}$ requires correction of only defocus and the two astigmatisms, which are the 
dominant aberrations. ${ }^{46}$ Although this would be of interest for future studies, it is beyond the scope of the current report. Additional potential improvements to the system could be the inclusion of optics to compensate for the longitudinal chromatic aberrations (b) introduced by the ey $\mathrm{e}^{47}$ and incorporation of computational/digital AO during postprocessing. ${ }^{48,49}$

\section{Conclusion}

We presented a multiscale SAO OCT capable of imaging in both standard OCT mode with 1.7-mm beam diameter incident on the cornea and high NA OCT mode with a 5.0-mm beam diameter incident on the cornea. Using the polarization properties of light and a single deformable element for changing the wavefront incident on a human eye, the system design was relatively simple and compact. The performance of the SAO algorithm was demonstrated within the isoplanatic patch by showing clear photoreceptor mosaic images and the spatial frequency distribution pattern forming Yellott's ring. The aberration correction was found to be effective in areas larger than the isoplanatic patch up to the FOV of $3 \operatorname{deg} \times 3 \operatorname{deg}(900 \mu \mathrm{m} \times 900 \mu \mathrm{m})$ for cone photoreceptor imaging in the parafovea. In addition, by applying a multiple BM-scanning protocol, SAO-OCT-A imaging performance with the two different imaging modes was demonstrated. From the OCT-A imaging results, we can visualize vasculature over the FOV of $15 \mathrm{deg} \times 15 \mathrm{deg}(4.5 \mathrm{~mm} \times 4.5 \mathrm{~mm})$ with standard OCT-A imaging, and more detailed vasculature and capillaries can be observed using high-resolution OCT-A imaging. The results indicate the strong potential for the clinical utility of the multiscale SAO-OCT-A system.

\section{Disclosures}

The authors have no relevant financial interests in this article and no potential conflicts of interest to disclose.

\section{Acknowledgments}

This research was supported by the Michael Smith Foundation for Health Research, Alzheimer Society Research Program, Natural Sciences and Engineering Research Council of Canada, Canadian Institutes of Health Research and National Health, and Brain Canada.

\section{References}

1. D. Huang et al., "Optical coherence tomography," Science 254(5035), 1178-1181 (1991).

2. W. Drexler and J. G. Fujimoto, "State-of-the-art retinal optical coherence tomography," Prog. Retinal Eye Res. 27(1), 45-88 (2008).

3. M. Wojtkowski, "New directions in ophthalmic optical coherence tomography," Optom. Vision Sci. 89(5), 524-542 (2012).

4. M. R. Hee et al., "Optical coherence tomography of the human retina," Arch Ophthalmol. 113(3), 325-332 (1995).

5. F. Roddier, Adaptive Optics in Astronomy, Cambridge University Press, Cambridge (1999).

6. D. T. Miller et al., "Adaptive optics and the eye (super resolution OCT)," Eye 25(3), 321-330 (2011).

7. B. Hermann et al., "Adaptive-optics ultrahigh-resolution optical coherence tomography," Opt. Lett. 29(18), 2142-2144 (2004).

8. R. J. Zawadzki et al., "Adaptive-optics optical coherence tomography for high-resolution and high-speed 3D retinal in vivo imaging," Opt. Express 13(21), 8532-8546 (2005).

9. D. X. Hammer et al., "Multimodal adaptive optics retinal imager: design and performance," J. Opt. Soc. Am. A 29(12), 2598-2607 (2012).

10. O. P. Kocaoglu et al., "Adaptive optics optical coherence tomography at 1 MHz," Biomed. Opt. Express 5(12), 4186-4200 (2014).
11. E. J. Fernandez et al., "Ultrahigh resolution optical coherence tomography and pancorrection for cellular imaging of the living human retina," Opt. Express 16(15), 11083-11094 (2008).

12. Y. Jian, R. J. Zawadzki, and M. V. Sarunic, "Adaptive optics optical coherence tomography for in vivo mouse retinal imaging," J. Biomed. Opt. 18(5), 056007 (2013).

13. R. S. Jonnal et al., "A review of adaptive optics optical coherence tomography: technical advances, scientific applications, and the future," Invest. Ophthalmol. Visual Sci. 57(9), OCT51-OCT68 (2016).

14. M. Pircher and R. J. J. Zawadzki, "Review of adaptive optics OCT (AOOCT): principles and applications for retinal imaging," Biomed. Opt. Express 8(5), 2536-2562 (2017).

15. Z. M. Dong et al., "Adaptive optics optical coherence tomography in glaucoma," Prog. Retinal Eye Res. 57, 76-88 (2017).

16. S. Marcos et al., "Vision science and adaptive optics, the state of the field," Vision Res. 132, 3-33 (2017).

17. M. Salas et al., "Visualization of micro-capillaries using optical coherence tomography angiography with and without adaptive optics," Biomed. Opt. Express 8(1), 207-222 (2017).

18. K. Kurokawa et al., "Three-dimensional retinal and choroidal capillary imaging by power Doppler optical coherence angiography with adaptive optics," Opt. Express 20(20), 22796-22812 (2012).

19. K. Kurokawa, Z. Liu, and D. T. Miller, "Adaptive optics optical coherence tomography angiography for morphometric analysis of choriocapillaris [Invited]," Biomed. Opt. Express 8(3), 1803-1822 (2017).

20. A. Dubra and Y. Sulai, "Reflective afocal broadband adaptive optics scanning ophthalmoscope," Biomed. Opt. Express 2(6), 1757-1768 (2011).

21. S.-H. Lee, J. S. Werner, and R. J. Zawadzki, "Improved visualization of outer retinal morphology with aberration cancelling reflective optical design for adaptive optics-optical coherence tomography," Biomed. Opt. Express 4(11), 2508-2517 (2013).

22. J. Zhang et al., "An adaptive optics imaging system designed for clinical use," Biomed. Opt. Express 6(6), 2120-2137 (2015).

23. Y. Jian et al., "Lens-based wavefront sensorless adaptive optics swept source OCT," Sci. Rep. 6, 27620 (2016).

24. Y.-J. Hong et al., "High-penetration swept source Doppler optical coherence angiography by fully numerical phase stabilization," Opt. Express 20(3), 2740-2760 (2012).

25. M. J. Ju et al., "Advanced multi-contrast Jones matrix optical coherence tomography for Doppler and polarization sensitive imaging," Opt. Express 21(16), 19412-19436 (2013).

26. F. C. Delori, R. H. Webb, and D. H. Sliney, "Maximum permissible exposures for ocular safety (ANSI 2000), with emphasis on ophthalmic devices," J. Opt. Soc. Am. A 24(5), 1250-1265 (2007).

27. A. Mariampillai et al., "Speckle variance detection of microvasculature using swept-source optical coherence tomography," Opt. Lett. 33(13), 1530-1532 (2008).

28. Y. Jian, K. Wong, and M. V. Sarunic, "Graphics processing unit accelerated optical coherence tomography processing at megahertz axial scan rate and high resolution video rate volumetric rendering.," J. Biomed. Opt. 18(2), 026002 (2013).

29. M. Cua et al., "Retinal optical coherence tomography at $1 \mu \mathrm{m}$ with dynamic focus control and axial motion tracking," J. Biomed. Opt. 21(2), 026007 (2016).

30. J. Xu et al., "Real-time acquisition and display of flow contrast using speckle variance optical coherence tomography in a graphics processing unit," J. Biomed. Opt. 19(2), 026001 (2014).

31. K. S. K. Wong et al., "In vivo imaging of human photoreceptor mosaic with wavefront sensorless adaptive optics optical coherence tomography," Biomed. Opt. Express 6(2), 580-590 (2015).

32. Y. Jian et al., "Wavefront sensorless adaptive optics optical coherence tomography for in vivo retinal imaging in mice," Biomed. Opt. Express 5(2), 547-559 (2014).

33. P. Bedggood et al., "Characteristics of the human isoplanatic patch and implications for adaptive optics retinal imaging," J. Biomed. Opt. 13, 024008 (2008).

34. J. I. Yellott, "Spectral analysis of spatial sampling by photoreceptors: topological disorder prevents aliasing," Vision Res. 22(9), 1205-1210 (1982).

35. S. H. Byeon et al., "New insights into the pathoanatomy of diabetic macular edema: angiographic patterns and optical coherence tomography," Retina 32(6), 1087-1099 (2012). 
36. N. Hasegawa et al., "New insights into microaneurysms in the deep capillary plexus detected by optical coherence tomography angiography in diabetic macular edema," Invest. Ophthalmol. Vision Sci. 57(9), OCT348-OCT348 (2016)

37. P. Henkind, "Radial peripapillary capillaries of the retina. I. Anatomy: human and comparative," Br. J. Ophthalmol. 51(2), 115-123 (1967).

38. P. Henkind, "Symposium on glaucoma: joint meeting with the national society for the prevention of blindness: new observations on the radial peripapillary capillaries," Invest. Ophthalmol. Vis. Sci. 6(2), 103-108 (1967).

39. P. K. Yu, S. J. Cringle, and D.-Y. Yu, "Correlation between the radial peripapillary capillaries and the retinal nerve fibre layer in the normal human retina," Exp. Eye Res. 129, 83-92 (2014).

40. Y. H. Kwon et al., "Primary open-angle glaucoma," N. Engl. J. Med. 360(11), 1113-1124 (2009).

41. R. F. Spaide, J. M. Klancnik, Jr., and M. J. Cooney, "Retinal vascular layers imaged by fluorescein angiography and optical coherence tomography angiography," JAMA Ophthalmol. 133(1), 45-50 (2015).

42. Z. Mammo et al., "Quantitative optical coherence tomography angiography of radial peripapillary capillaries in glaucoma, glaucoma suspect, and normal eyes," Am. J. Ophthalmol. 170, 41-49 (2016).
43. M. Heisler et al., "Strip-based registration of serially acquired optical coherence tomography angiography," J. Biomed. Opt. 22(3), 036007 (2017).

44. P. Prentasic et al., "Segmentation of the foveal microvasculature using deep learning networks," J. Biomed. Opt. 21(12), 075008 (2016).

45. M. Reddikumar et al., "Optical coherence tomography with a $2.8-\mathrm{mm}$ beam diameter and sensorless defocus and astigmatism correction," J. Biomed. Opt. 22(2), 026005 (2017).

46. J. F. Castejón-Mochón et al., "Ocular wave-front aberration statistics in a normal young population," Vision Res. 42(13), 1611-1617 (2002).

47. R. J. Zawadzki et al., "Ultrahigh-resolution optical coherence tomography with monochromatic and chromatic aberration correction," Opt. Express 16(11), 8126-8143 (2008).

48. Y.-Z. Liu et al., "Computational optical coherence tomography [Invited]," Biomed. Opt. Express 8(3), 1549-1574 (2017).

49. L. Ginner et al., "Noniterative digital aberration correction for cellular resolution retinal optical coherence tomography in vivo," Optica 4(8), 924-931 (2017).

Biographies for the authors are not available. 